\title{
Optimum Design of the Lower Plate in Die Cutting Machine Based on ANSYS
}

\author{
Wang Jinlong \\ College of Civil Engineering and Architecture of Weifang University, \\ Weifang 261061, China \\ wfxyjinlong@163.com
}

\begin{abstract}
In order to study the MWB1450 type die cutting machine, geometry entity model and finite element model of lower plate were established by ANSYS software, and stress analysis, displacement analysis and fatigue life prediction of the lower plate are proceeded. Based on this, puts forward the measures in optimizing the structure of the lower plate, and making reasonable design and improvement to the lower plate, the optimization of lightweight, high strength and durability have been achieved.
\end{abstract}

Keywords: Die Cutting Machine ; Finite Element Model ; Optimum Design; Fatigue Analysis

ANSYS is fusion structure, thermal, fluid, electromagnetic field, acoustic field and the coupling field analysis in the integration of large general finite element analysis software, has been widely used in machinery, transportation, military, electronics, biomedical, water conservancy, petroleum chemical industry, energy, aerospace and many other fields. Since 1970, John Swanson developed the ANSYS software, then ANSYS software with its powerful before and after processing function get the favour of many researchers and engineers. Especially in the aspect of structure analysis, ANSYS provides not only the linear calculation, also includes such as plastic, creep, expansion, large deformation, large strain and nonlinear calculation technology of contact analysis. For 40 years, it continuously absorb the new calculation method and computing technology, with the increase of interaction, to simplify generation of model and evaluation of results, especially its powerful post-processing function, which greatly simplifies data processing and result analysis after finite element analysis, to some extent, reduces stress analysis and design time and shortens design cycle.Due to its advancement and accuracy in structure analysis, ANSYS software is always preferred in structural analysis[1].

\section{Die Cutting Machine Introduction}

As one of post-press processing equipments which are automation and high technology, the automatic die cutting machine is widely used currently. Owing to much pressure are needed during the die-cutting process, so most of die cutting machine manufacturers adopt the double toggle lever mechanism to improve the press structure. The maximum die cutting pressure of MWB1450 is 300 tons. Die cutting pressure is a kind of contact force, its size changes with the platform of the displacement and the movement rate of the platform. The larger the displacement which is squeezed by the top and bottom platforms, the greater the die shear is; the higher die cutting machine working speed is, the higher the speed of the moving platform and the impact force of the fixed platform are. Because of the nonlinear relative displacement between the contact force and contact parts, so the displacement between the die cutting pressure and the moving platform is nonlinear. And in the work schedule, die-cutting pressure may not be always uniformly distributed load. But in the practice of die cutting indentation process, after the version we should regulate 
firstly to make the pressure of the layout of the cutter line and steel wire to achieve uniform respectively, then formally put the die cutting machine into use after a die cutting test. During the analysis of the die cutting pressure, we can approximately regard the maximum pressure as uniformly distributed load. After the bottom platforms entered, die cutting pressure can approximately regard as the slowly increasing static load. 300 tons uniform die cutting pressure is applied to the moving platform surface.

\section{Intensity Analysis of the Lower Plate in Die Cutting Machine}

\subsection{Establishment of Geometry Model}

According to engineering drawing, the 3-D geometric model of the lower plate was established, As is shown in Figure 2.

To save computational resources, both make the necessary simplification to the lower plate model in ANSYS, and ensure the accuracy of the calculation, greatly makes the finite element model is in conformity with the practical engineering problems. For this purpose ,simplified off the round corner, chamfer, screw holes of the lower plate, etc.
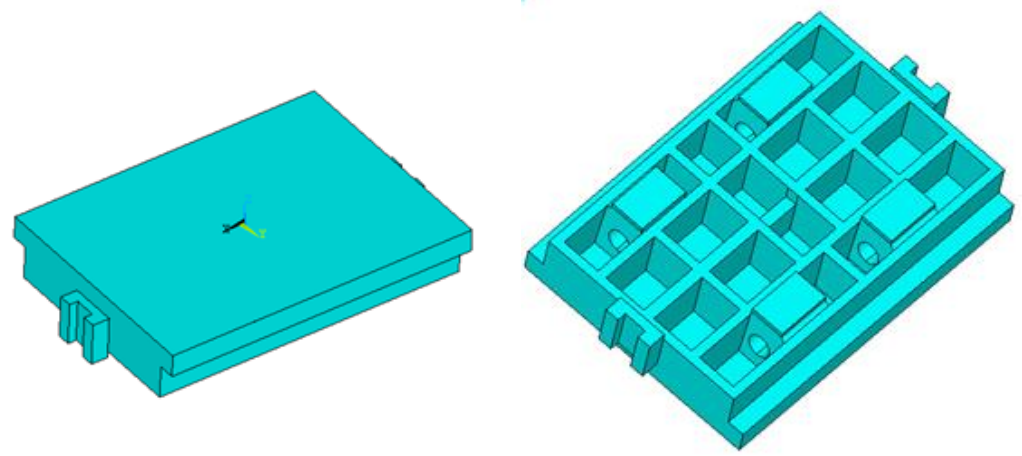

Figure 1. 3-D Geometric Model of the Lower Plate

\subsection{Material Parameters of the Lower Plate}

The material Parameters of the lower plate are shown in Table 1.

Table 1. Material Parameters

\begin{tabular}{ccccc}
\hline materials & MOE/GPa & PR & $\begin{array}{c}\text { strength of } \\
\text { extension/MPa }\end{array}$ & $\begin{array}{c}\text { permissible } \\
\text { stress/MPa }\end{array}$ \\
\hline QT500-7 & 173 & 0.3 & 500 & 334 \\
\hline
\end{tabular}

\subsection{Establishment of the Finite Element Model}

The 3-D finite element model of the lower plate is shown in Figure 2. 


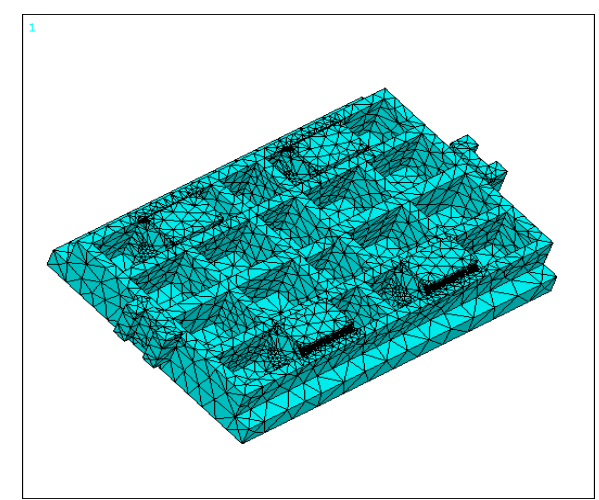

Figure 2. Finite Element Model of the Lower Plate

\subsection{Applying Constraints}

When the lower plate contact with the upper plate, we take it as a fixed platform by reason of it bearing reaction force, and die cutting pressure is applied on its surface, all constraints is put on its support.

\subsection{Strength Calculation}

After determining the material, constraints, and meshing, the stress and displacement for the lower plate in 300 tons of die cutting pressure are obtained by the finite element calculation, as shown in Figure 3 and 4.
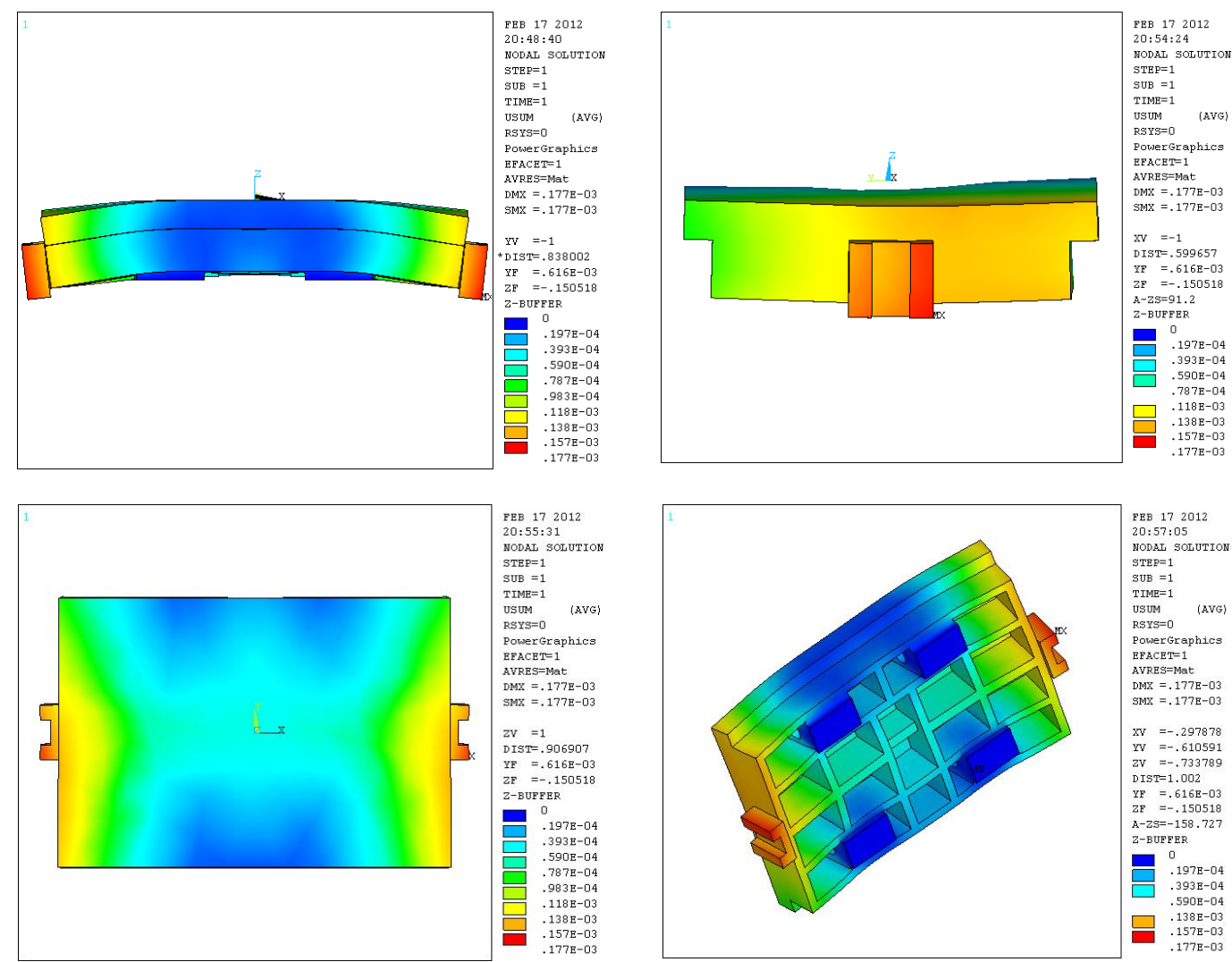

Figure 3. Displacement Diagram of the Lower Plate 

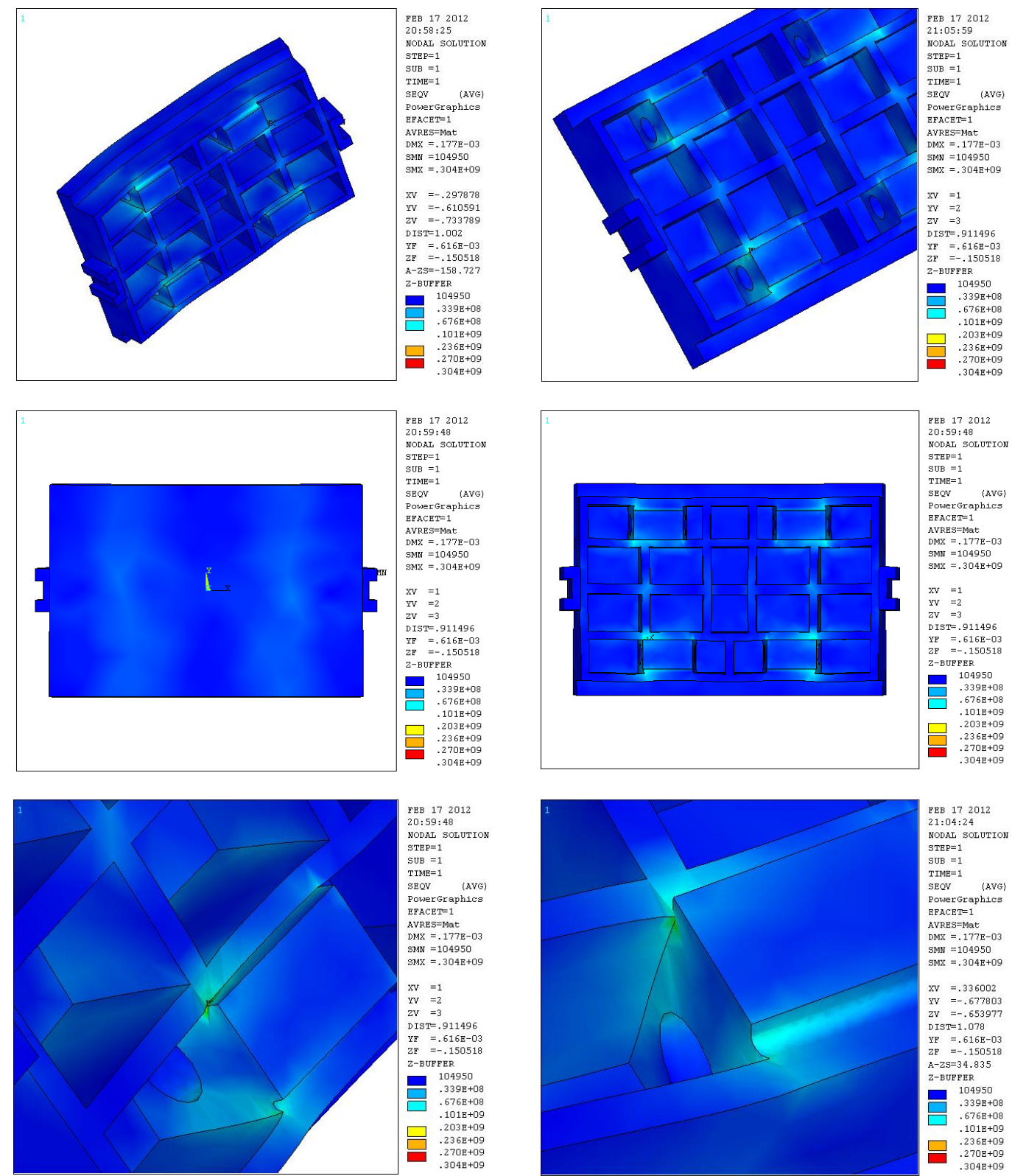

Figure 4. Effective Stress Nephogram of the Lower Plate

The Figure 3 and 4 show that the maximum displacement appeared in the two sides slide rail of the lower plate, its maximum displacement value is $0.177 \mathrm{~mm}$, which could meet the precision demand of the die cutting machine. The maximum stress appeared in the corner of the lower plate supporting system, it mainly passed from toggle force and supported the lower platform, its maximum stress value is $304 \mathrm{MPa}$, which is less than the allowable stress $334 \mathrm{MPa}$, From its stiffness and strength, the structure is safe and reliable.

To sum up, the stiffness and strength of the lower plate is safe and reliable before optimization, but it should be pay attention to the stress concentration phenomenon of the corner of the supporting system. 


\section{Optimization of the Lower Plate}

\subsection{Optimization Theory}

Through ANSYS software, the finite element method and optimization technique are organically combined to provide convenience for designers. In the optimization ,the finite element method is one of the important methods.The basic principle of optimization problems is through the establishment of optimization models, using various optimization methods, by meeting the design requirements under iterative calculations to obtain extreme value of the objective function and optimal design scheme [3]. The mathematical model of optimization problem is expressed as [4]:

$$
\begin{aligned}
& \operatorname{Min} F(X)=\left(x_{1}, x_{2}, \ldots \ldots, x_{N}\right) \\
& \text { Find } X=\left(x_{1}, x_{2}, \ldots \ldots, x_{N}\right)^{T} \in R \\
& \text { s.t.g } g_{i}(X)=g\left(x_{1}, x_{2}, \ldots \ldots, x_{m}\right) \leq 0 \quad i=(1,2, \ldots, m) \\
& h_{j}(X)=g\left(x_{1}, x_{2} \ldots \ldots x_{n}\right)=0 \quad j=(1,2, \ldots \ldots, n)
\end{aligned}
$$

Here, $F(X)$ is the objective function, it is the function of design variables that is used to evaluate good and bad of design proposal; $g_{i}(X), h_{j}(X)$ are the constraint condition; $m, n$ are the number of state variables $x$ is the design vectors.

Optimized variables are the basic variables in the process of optimization design, including the design variables, state variables and objective function. The Design variables are independent variables in optimization design, usually including geometry (such as area, width and height, etc.), material, load and constraint location, etc. The optimized results are obtained by changing design variable values. Every design variable has upper and lower limits, which define the range of the design parameters. State variables can restraint the value of design variables, usually including internal force, bending moment and stress and displacement and so on. State variable, also known as "the dependent variable", is a function of the design variables. Only the state variables accord with the prescribed restricted conditions, the design can be reasonable, thus to realize the optimization design. Objective function refers to the optimized design value, usually including structure weight, size(such as thickness), shape(such as the transition fillet radius), support location and manufacturing cost, etc. Objective function must be a function of design variables, that is to say, the change of design variables will lead to the change of the objective function value[5].

Optimization method is one of traditional methods which can make a single function attain the minimum value under the restricted conditions, including the sub-problem method and first-order method. The essence of the sub-problem method adopts the least squares approximation method to obtain a function that fits the solution space and calculate the extreme value of the objection function. The sub-problem method is widely used and not easy to fall into local extreme points, but it has not high precision, so it mainly is used for coarse optimization phase. Based on the sensitive degree of the objective function for design variables, the first-order method is more suitable for the accurate analysis of the optimization.

\subsection{Problem Description of the Lower Plate}

The upper surface thickness of the lower plate has a range from 0 to 0.05 , its safety coefficient is 1.5. Optimization design requirements:under the premise of the lower plate satisfying the demands of a given stiffness and strength, it achieved the minimum weight by optimizing the upper surface thickness of the lower plate. 


\subsection{Mathematical Model of Optimal Design}

Mathematical model consists of the objective function and constraint conditions. According to the requirements, under the premise of the lower plate not do too much change, to optimize the upper surface thickness ${ }_{H}$ of the lower plate,and take ${ }_{H}$ as the design variables, take the equivalent stress $\sigma$ and resultant displacement $D$ of the lower plate as constraints, take the weight $f(x)$ of the lower plate as objective function.

In conclusion, the mathematical model of optimization design for the lower plate is as follows:

$$
\begin{aligned}
& W_{t}=\min f(x) \\
& X=[H], \sigma \leq[\sigma] \\
& 0 \leq H \leq 0.05,0 \leq D \leq 0.002
\end{aligned}
$$

\subsection{Optimal Computation of the Lower Plate}

After confirmed the material, constraints, the lower plate was optimized. The optimization results and process as shown in Figure 5.
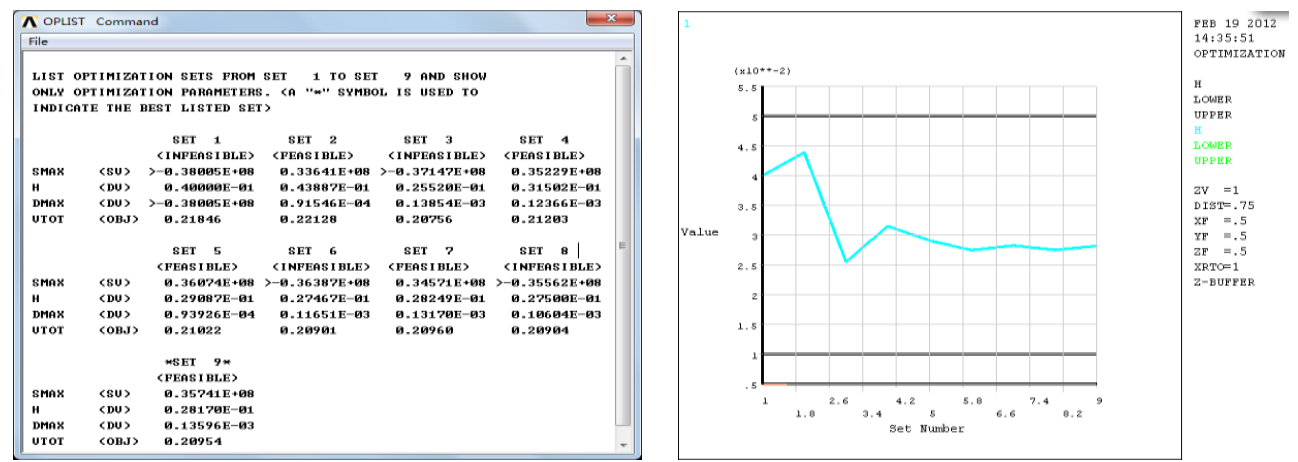

Figure 5. Optimization Results and Process

From Figure 5, the optimization results of $H$ value tend to uniformity after the fourth iteration. According to the optimal target function, that is the minimum weight principle of the lower plate, the final optimization results should choose the ninth calculation results in the figure, the value of $H$ is $28.17 \mathrm{~mm}$, as the objective function dropped $8 \%$, the optimization effect is obvious.

On the basis of optimization, we recalculate the optimized model when the $H$ value is $30 \mathrm{~mm}$, collected date again in the post-processing and re-analysed.

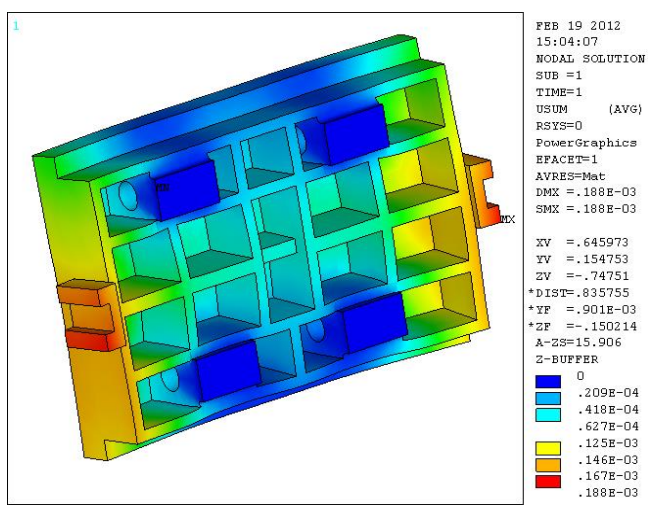

Figure 6. Displacement Diagram of the Lower Plate

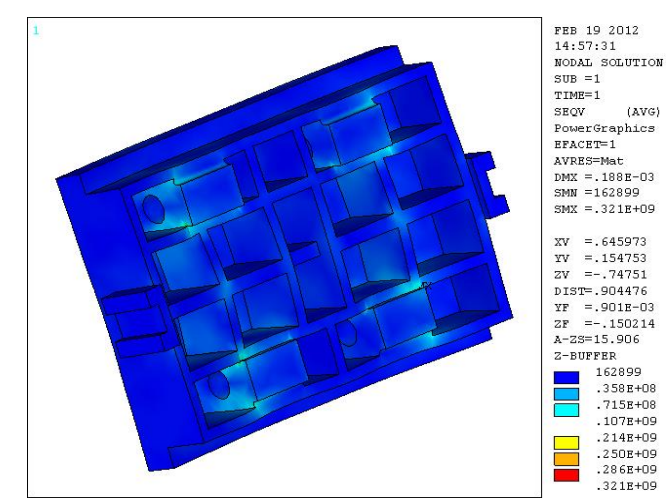

Figure 7. Effective Stress Nephogram of the Lower Plate 
From Figures 6 and 7, When applying 300 tons of die shear on the surface of the lower plate, the maximum effective stress value is $321 \mathrm{Mpa}$ in the Angle of supporting point for the lower plate, its value is less than the allowable stress $334 \mathrm{MPa}$, the maximal displacement is $0.188 \mathrm{~mm}$. The results showed that the strength and stiffness of the lower plate meets the design requirement.

\section{Fatigue Analysis}

\subsection{Fatigue Analysis Theory of ANSYS}

Fatigue is one of the fracture damage phenomenon of structure under the action of repeated load below static strength. Fatigue analysis adopts classic Miner linear cumulative damage theory in ANSYS [6]. See the generation of fatigue damage as a deterministic events, the fatigue damage will occurs if fatigue stress amplitude is greater than fatigue limit, it's the safer using this method to calculate the fatigue life.

If the component cycle to the destruction of life is $N$ under the action of a constant stress $S$, then defining its damage at $\mathrm{N}$ cycle as follow:

$$
D=n / N
$$

Obviously, under the action of a constant stress $s$, if $n=0$, then $D=0$, At this point, the component were undamaged by fatigue. If $n=N$, then $D=1$, At this point, the component were damaged by fatigue[7].

Damage of components that experienced $n_{i}$ cycles is $D_{i}=n_{i} / N_{i}$ under the action of a stress $S_{i}$. Damage of components that experienced $n_{i}$ cycles under the action of $k$ stress $s_{i}$, defining its total damage called fatigue life using coefficient in ANSYS as follows:

Failure criterion as follows:

$$
D=\sum_{i=1}^{k} D_{i}=\sum_{i=1}^{k} n_{i} / N_{i}
$$

$$
D=\sum_{i=1}^{k} D_{i}=1
$$

Here, $n_{i}$ is the number of loops under the action of stress $S_{i} ; N_{i}$ is the component cycle to the destruction of life under the action of stress $s_{i}$, that be determined by the S-N curve.

\subsection{Fatigue Analysis of the Lower Plate}

The result of FEM showed that the maximum stress was located in the corners location of the lower plate, and prediction results agrees. The local area of the largest stress values are to endure random dynamic loads for practical use, probably prone to cracks, then extended rupture, and lead to failure, it is necessary to further study on the fatigue strength analysis. This article utilizes ANSYS fatigue analysis module to explore and estimate cumulative use factor of the fatigue life for the lower plate [8]. In ANSYS software, the unit life consumption factor is determined by the stress results of postprocessing[9]. After the completion of the above, to determine stress concentration areas of the stress nephogram, this position is prone to fatigue damage parts, so choose it near nodes as fatigue analysis object.

Adopting the S-N curve of ductile iron as the input data of analysis [10], as shown in Figure 8. The dangerous point of the stress maximum value and the loads of events are defined in fatigue analysis, which is a stress state of the event, and set the total equivalent cyclic number of the event. 


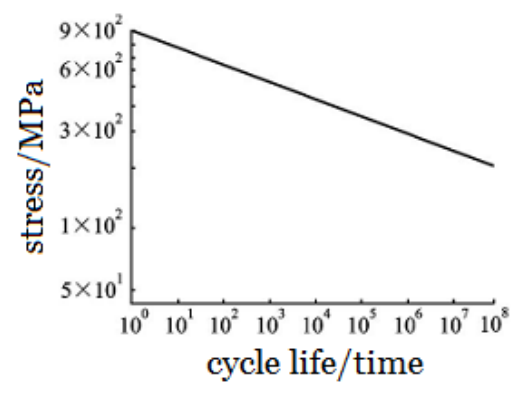

Figure 8. S-N Curve of Ductile Iron

The fatigue life of the lower plate was analyzed by ANSYS software, and its cumulative use coefficient was obtained, its value is $0.48<1$. The result shows that the fatigue life of the lower plate satisfy the design requirement, the calculation results as shown in figure 9 . The process can be reformulated with more detail as follows .

(1) The determination of fatigue parameters for the lower plate [11]

In fatigue calculation: 1 node location, 1 event, 2 loads per event, namely the maximum working pressure and minimum working pressure.

(2)The material fatigue property was defined by the S-N curve, namely the relation curve of the stress amplitude $\left(S_{\max }-S_{\min }\right) / 2$ and the fatigue cycles times.

(3) The maximum effective stress was located in the corners location of the lower plate, for the fatigue failure of the most dangerous point, the cycle for pulsating cycle, the maximum stress is $321 \mathrm{MPa}$, so alternating stress amplitude is $160.5 \mathrm{MPa}$. The service life of the lower plate is calculated in 15 years, then its cycle times is $4.8 \mathrm{e} 8$.

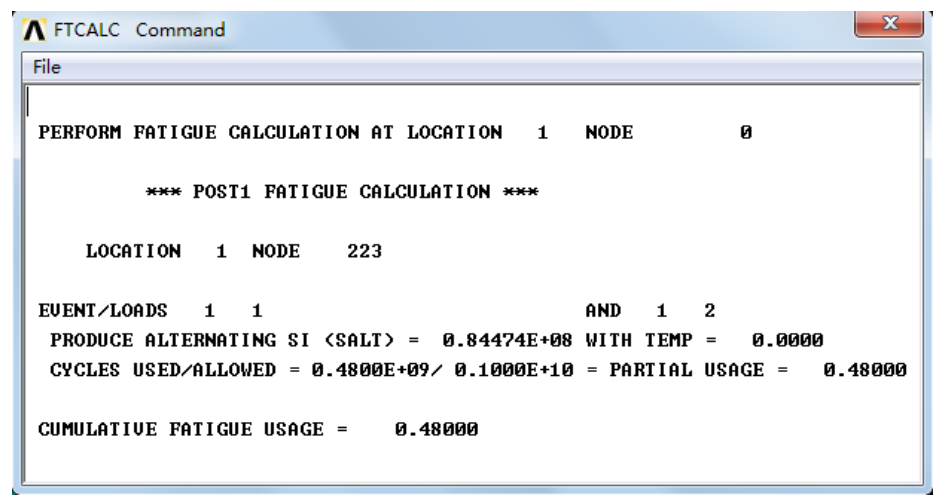

Figure 9. Fatigue Calculation Results

\section{Conclusions}

(1)Taking the lower plate of MWB1450 type die-cutting machine as study object, a para-metric model has been established with finite element ANSYS, and the thickness of the lower plate has been also optimized, its $30 \mathrm{~mm}$ reasonable thickness has been obtained. Moreover, the stress analysis for the optimized model has been made, to obtain maximum stress was $321 \mathrm{MPa}$, the maximal displacement was $0.188 \mathrm{~mm}$, both the strength and rigidity of the lower plate were suitable for design requirements.

(2)The maximum effective stress of the lower plate was obtained through finite element analysis, and was located in the corners location of the lower plate, its maximum effective stress is $321 \mathrm{MPa}$.

(3)Taking the lower plate of MWB1450 type die-cutting machine as study object a finite element model has been established with ANSYS, Then, the fatigue strength 
analysis is carried out. Through analysis shows that the cumulative use coefficient of the lower plate is $0.48<1$, the Angle of the supporting points parts meet the requirements of fatigue strength, the lower plate is safe, but we still need to pay attention to at the Angle of the supporting point, and should adopt the method of avoiding stress concentration to improve the fatigue life of the lower plate.

(4)Analysis results shows that the fatigue analysis functions of ANSYS structural fatigue analysis is feasible, the method is simple, and avoids a large number of trials, and save the resources, it is the combination of finite element theory and the theory of fatigue analysis, and provides a new method of fatigue analysis for complex structure.

\section{Acknowledgements}

The work in this paper has been supported by funding from Outstanding Young and middle-aged scientists Foundation of Shandong(BS2010CL046).

\section{References}

[1] W. Jin-long, W. Qing-ming and W. Wei-zhang," ANSYS 12.0 Finite Element Analysis and Example", Beijing: China Machine Press, (2010), pp. 5-6.

[2] C. Gang-hu, C. San-zhong and Z. Xuan-sheng, Journal of Xi'an University of Techonlogy ,vol. 22, no. 3, (2006), p p. 249-252.

[3] Z. Chao-hui, " ANSYS 11.0 Structural Analysis Engineering Application Examples ", Beijing: China Machine Press, (2008), pp. 560-582.

[4] S. Jing-min, L. Ying-chun, "Mechanical Optimum Design", Beijing: Mechanic Industry Press, (2006)

[5] F. Shi-jie, Q. Yao-guang, "Mechanical Optimum Design", Beijing: Mechanic Industry Press, (2003).

[6] L. Wei-xin, "Mechanism reliability design", Beijing:Press of Tsinghua University, (1996), pp. 221-278.

[7] W. Zhang,K. J. Miller,"A Study of Cumulative Fatigue Damage Under Variable Loading-mode Conditions", Fatigue Fract Engng Mater Struct, vol. 19, no. 2/3, (1996), pp. 229-239.

[8] L. Guo-qing and W. Mao-ting, "Light Industry Machinery", vol. 29, no. 2, (2011), pp. 116-119.

[9] Y. Wei-xing," Structure Fatigue analysis", Beijing: National Defence Industry Press, (2006), pp. 34-73.

[10] H. You-shi, Z.Ai-guo, Q. Gui-an, acta metallurgica sinica, vol. 45, no. 7, (2007), pp. 769-780.

[11] W. Qing-feng, H. Li-dong, Thermal Power Generation, vol. 29, no. 1, (2010), pp. 32-35.

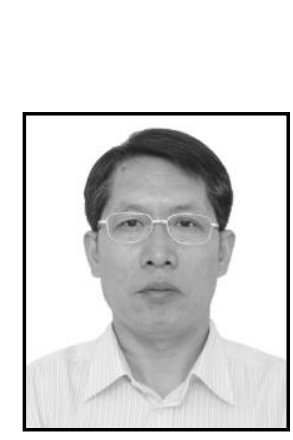

\section{Author}

Jinlong Wang, he received his doctor's degree from China University of Petroleum. He is currently an associate professor in College of Civil Engineering and Architecture, Weifang University, China and he has published over 36 research papers in scholarly journals and international conferences. 
International Journal of Control and Automation Vol. 8, No. 7 (2015) 